\title{
RESEARCH NOTE: permeability control on the relationship between nuclear magnetic resonance (NMR) measured porosity and routine core porosity
}

\author{
Mohammed A. Aqel ${ }^{1}$ \\ Received: 6 January 2015 / Accepted: 20 January 2016 / Published online: 21 April 2016 \\ (C) The Author(s) 2016. This article is published with open access at Springerlink.com
}

\begin{abstract}
A new criterium is proposed as a hypothesis that gives a dividing line between conventional and unconventional reservoir rocks from the permeability point of view. For rocks with higher permeability, the laboratory porosity values are higher than the NMR porosities. For rocks of lower permeability, the laboratory porosity values are lower compared to NMR porosities. This tendency of permeability to control the relation between porosities measured by two techniques is noticed in published data collected from different literature sources.
\end{abstract}

Keywords Unconventional reservoir rocks · Permeability · NMR porosity $\cdot$ Routine porosity

\section{Introduction}

NMR and laboratory porosity measurements were collected from published papers for carbonate rock plugs. Three sets of data were used in this study, two of which are from the same source: (1) Twenty-seven (27) carbonate core plugs analyzed by low-field NMR and conventional core analysis (Chang et al. 1997), (2) a total of 35 core plugs selected from off-shore, carbonate, oil-producing formations (the Glorieta carbonates in West Texas): Field A. (Lyne et al. 1996), and (3) a total of 29 core plugs selected from off-shore, carbonate, oil-producing

Mohammed A. Aqel

aqeel15@hotmail.com

1 King Fahd University of Petroleum \& Minerals, Dhahran, Saudi Arabia formations (the Clearfork carbonates in West Texas): Field N. (Lyne et al. 1996).

\section{Porosity and permeability procedures}

Porosity can be determined by different lab procedures. Porosity is determined by using helium-gas expansion as described by Boyle's law (Kazimierz et al, 2004) where in a gaseous system at a given temperature, the product of absolute pressure and volume is constant. Also, porosity is calculated by using saturation method where the pore volume is determined by dividing the difference in weight between the saturated sample and the dry sample by the known density of the saturating fluid.

And by applying Archie's laws on laboratory-measured electrical properties (i.e., resistivity, formation resistivity factor, and cementation exponent), porosity is calculated (Efnik et al, 2006). In NMR measurements, core plug measurements provide important details about the variations in the pore size, and free/bound fluid volumes (Moss, 2004).

Using Darcy's law, permeability is calculated from fluid flow rate, cross-sectional area of the core sample, differential pressure (between upstream and downstream pressures), viscosity of flowing fluid, and length of the core sample.

For the first set (Chang et al, 1997), plugs were cleaned for laboratory porosity measurements by hydrostatic weighing in toluene and for Klinkenberg-corrected air permeability measurements. Then, the samples were saturated by brine for NMR porosity measurements. Similarly, the second set of plugs (Lyne et al, 1996) were cleaned and resaturated with brine. Laboratory (conventional) porosity and permeability measurements were conducted by following the Archimedes principle and the Klinkenberg correction, respectively (Tanikawa and Shimamoto, 2006). The NMR porosity values 
Fig. 1 Tendency of permeability control on porosities' relationship for $75 \%$ of the data: Permeability versus relative error between NMR and Lab porosities (Data taken from Chang et al. 1997)

Fig. 2 Tendency of permeability control on porosities' relationship for $74 \%$ of the data for Field A: Permeability versus relative error between NMR and Lab porosities (Data taken from Lyne et al. 1996). Note that this group can be divided into two sets (red squares with higher permeability values and blue diamonds with lower permeability values) having the same relationship

Fig. 3 Tendency of permeability control on porosities' relationship for $100 \%$ of the data for Field N Permeability versus relative error between NMR and Lab porosities
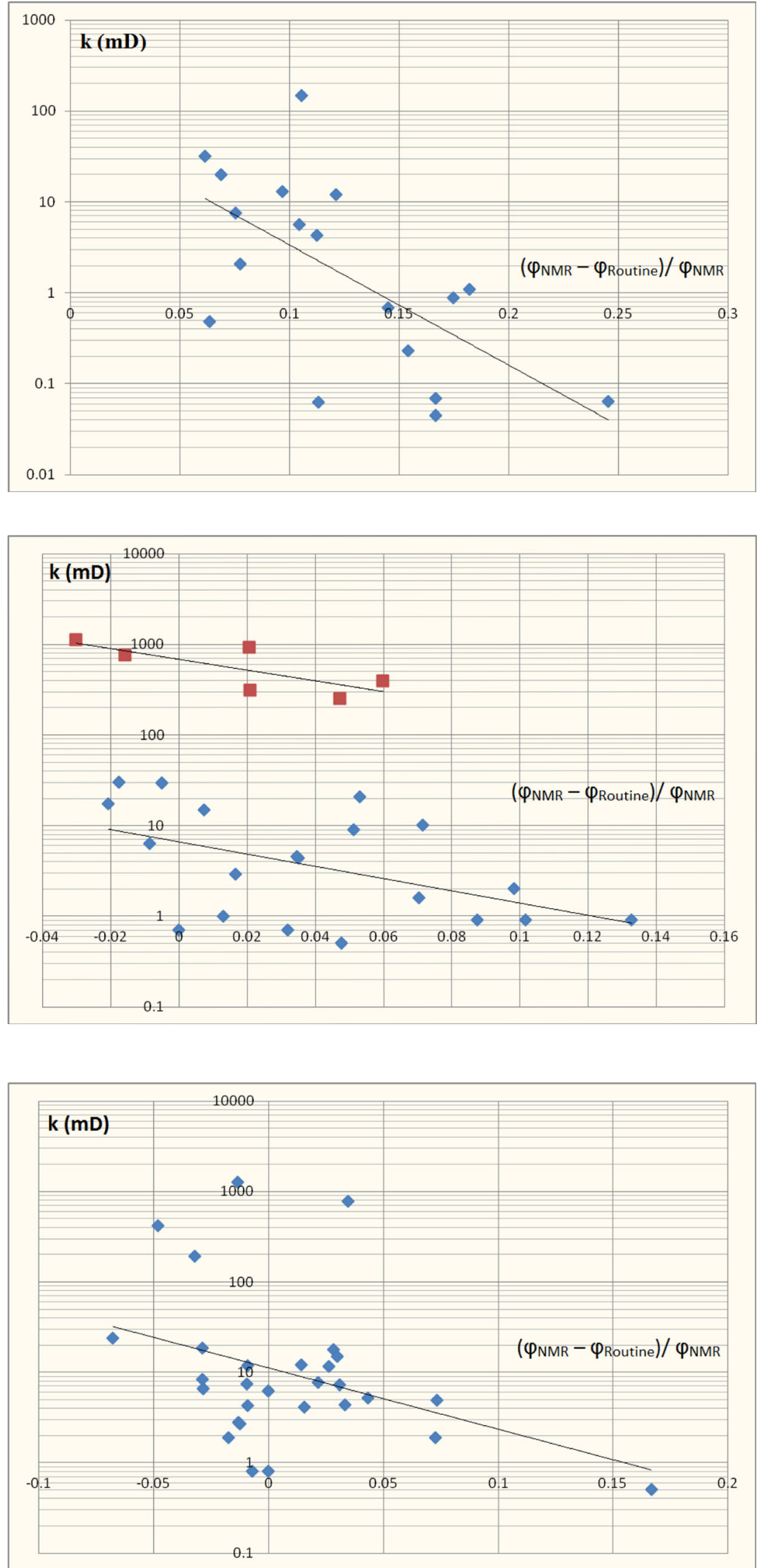
Fig. 4 All data points plotted in one graph showing the tendency

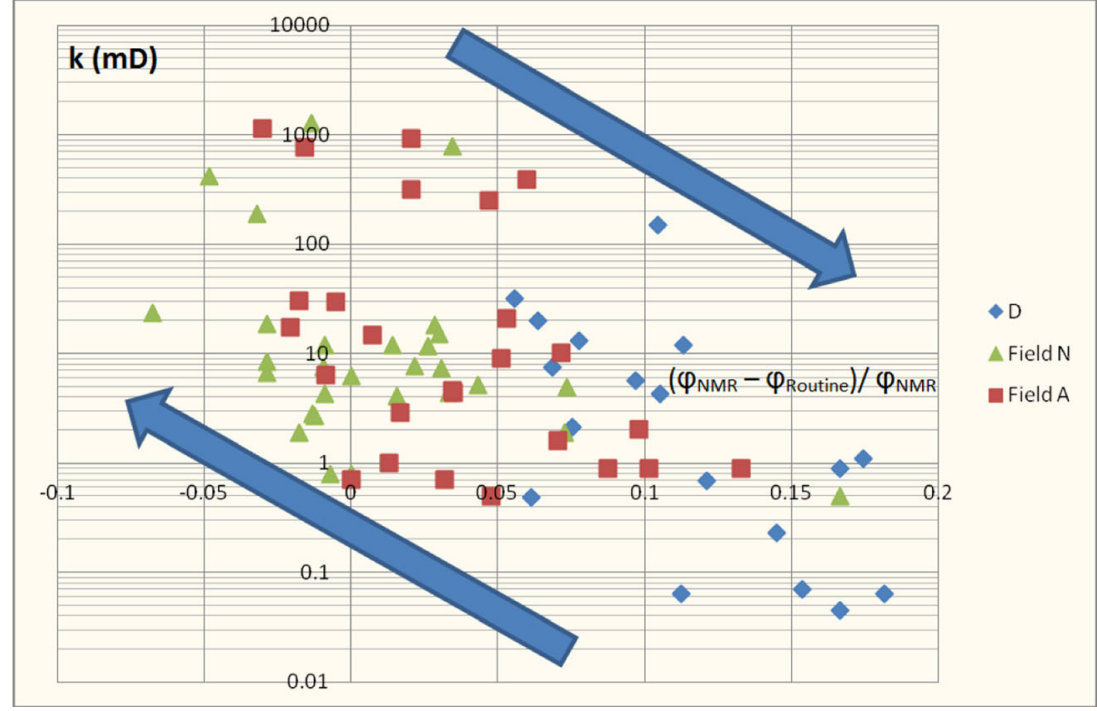

were calculated directly from the NMR signals as they are proportional to the volume of fluids in the pore systems.

\section{Interpretation}

Inverse relations between the relative errors of porosities and permeability values are shown in Figs. 1, 2, and 3. When NMR porosity is greater than conventional plug porosity, the permeability value is expected to be low and vice versa.

\section{Hypothesis}

The measured permeability values were plotted against the relative error between "NMR" porosity and "LAB" porosity (Figs. 1-3).

The permeability controls the relation between NMRmeasured porosity and conventional laboratory-measured porosity according to the best fit relationship:

$y=a e^{b x}$

where $y$ is the permeability and $x$ is the relative error of porosity measurements.

I assume, as a work hypothesis, that for higher permeability values, the laboratory porosity values are higher than the NMR porosities (Fig. 4). And, for lower values of permeability, the laboratory porosity values are lower compared to NMR porosities. It seems likely that this observation would establish a discriminatory threshold between conventional and tight rocks. Further research is needed to involve in this study the three classic logderived (density, neutron, and sonic) porosities and their relation with the NMR porosity.

\section{Conclusion}

The proposed trend - if verified - could delineate the boundary between conventional and unconventional rocks from the permeability point of view. However, more data are needed for a better definition of the control parameters.

Acknowledgments The author thanks Professor Gabor Korvin (King Fahd University of Petroleum and Minerals, KFUPM, Saudi Arabia) for his valuable comments and suggestions which improved the original manuscript.

Open Access This article is distributed under the terms of the Creative Commons Attribution 4.0 International License (http:// creativecommons.org/licenses/by/4.0/), which permits unrestricted use, distribution, and reproduction in any medium, provided you give appropriate credit to the original author(s) and the source, provide a link to the Creative Commons license, and indicate if changes were made.

\section{References}

Chang D, Vinega H, Morriss C, Straley C (1997) Effective porosity, producible fluid, and permeability in carbonates from NMR Logging. The Log Analyst, March.-April, 60-72

Efnik M, Dernaika M, Kalam M (2006) Evaluation of Water Saturation from Laboratory to Logs. The International Symposium of the Society of Core Analysts. SCA2006-56 p 1-6

Kazimierz T, Jacek T, Stanisław R (2004) Evaluation of Rock Porosity Measurement Accuracy with a Helium Porosimeter. Acta Montan Slovaca 9(3), p 316-318

Lyne A, Vaflni G, Ghilardotti G (1996) Determination of petrophysical properties of carbonate rocks by NMR relaxometry. Society of Petroleum Engineers, (SPE 36852), 331-339

Moss A (2004) NMR Core Plug Measurements to Compliment SCAL studies. The International Symposium of the Society of Core Analysts. SCA2004-47 p 1-6

Tanikawa W, Shimamoto T (2006) Klinkenberg effect for gas permeability and its comparison to water permeability for porous sedimentary rocks. Hydrol Earth Syst Sci Discuss 3, 1315-1338 